Manuelle Medizin 2012 · 50:424-424

DOI 10.1007/s00337-012-0970-z

Online publiziert: 25. Oktober 2012

๑) Springer-Verlag Berlin Heidelberg 2012

\section{H.M. Bullinger}

Dortmund

\section{KISS und die Öffnung verschlossener Türen}

\section{Leserbrief zu}

Heymann W von (2012) „,Tonusasymmetriesyndrom" und ,sensomotorische Dyskybernese". Manuelle Med 50:285-288

Liebe Kolleginnen und Kollegen,

Jubiläumszeitschriften enthalten meist einen versöhnlichen Rückblick auf die Entwicklung eines Fachgebiets und die Zeitschrift selbst. Ältere Leser erhalten vielleicht ein berufsbegleitendes Resümee. Für die Idee, ein Heft herauszugeben, das die Erinnerungen an die Anfänge der Manualmedizin mit ihren Gründungsvätern und Protagonisten auch für die jüngere Leserschaft aufzeigt, bin ich sehr dankbar.

Peinlich berührt und überrascht war ich hingegen über den Kommentar des Vorsitzenden der MWE mit seinem Beitrag „Tonusasymmetriesyndrom und sensomotorische Dyskybernese“. Gespannt auf den fachlichen Sprung zwischen Gutmanns „cervicaldiencephal-statischem Syndrom" und dem Leitthema wurde der Leser enttäuscht und dennoch überrascht. Während die Wertung der aktuellen Konzepte in der manuellen Säuglingsbehandlung in „seminarspezifischer Färbung“ gesehen werden kann, so war die polemisch gehaltene Wortwahl und Intention deutlich unangemessen, deplatziert und auch fachlich wie historisch nicht korrekt.

Nur am Rande sei erwähnt, dass der Begriff KISS schon in den Jahren 1989/90 entstand und in einer kinderärztlichen Zeitschrift mit den Möglichkeiten und Grenzen der manuellen Medizin erstmals veröffentlicht wurde unter Bezugnahme auf Gutmanns grundlegende Arbeiten.
Die „Abrechnung“ mit dem Kollegen Biedermann gipfelt letztlich in dem Verweis auf die fehlende Unterzeichnung eines Konsenspapiers, das von ihm weder erarbeitet noch ihm vorgelegt wurde.

Dies in einer Zeit, in der es umso wichtiger wird, dass sich jene Kollegen, die mit der manuellen Behandlung bei Säuglingen und Kleinkindern beschäftigt sind, konzeptübergreifend mehr dem Gemeinsamen und Verbindenden widmen. Von Heymanns Kommentar erscheint in einer Zeit, in der juristische Auseinandersetzungen um medizinische Behandlungskonzepte den Praxisalltag begleiten. Ausgerechnet in einer Zeit, in der alle Energie gebündelt werden sollte, um überzeugende Studiendesigns zu schaffen, die unser aller Erfahrung und Überzeugung Rechnung tragen können, wird lieber zu „schwerem Gerät" gegriffen, um die alten Gräben, die sich langsam füllen, erneut zu vertiefen. Wie weit muss man sich vom Praxisalltag entfernt haben?

Die Art und Weise, wie in dem Kommentar mit der Lebensleistung von Heiner Biedermann umgegangen wird, kann nur verständnisloses Kopfschütteln auslösen. Wer ihn kennt weiß, dass er unbequem ist. Dennoch ist es immer die kollegiale hartnäckige Disputation, die die Medizin voranbringt. Heiner Biedermann zu diskreditieren und sein Verdienst um die manuelle Behandlung von Kindern in über 30 Jahren so zu reflektieren und herabzuwürdigen, dafür habe ich kein Verständnis. Das „populistische KISS“ hat es uns ermöglicht, sowohl mit Eltern als auch unter uns Ärzten einen Symptomenkomplex zu kommunizieren und zu erörtern. Fachkundige wissen, dass es Bestrebungen gab, „ähnlich“ griffige Namen zu eta- blieren. Selbige Fachkundige wissen auch, dass viele Arbeiten Gutmanns und vor allem seine Buchreihe in den 1980er Jahren unter wesentlicher Mitwirkung von Heiner Biedermann entstanden sind. Die erfolgreiche Behandlung von KISS und die Öffnung zahlreicher bis dahin für die manuelle Kinderbehandlung verschlossener Türen hätten Biedermanns Lehrer und Mentor wahrscheinlich stolz gemacht, diese geniale Idee sollten wir deshalb neidlos anerkennen.

Dieser Kommentar ist Wasser auf die Mühlen derer, die der manuellen Kinderbehandlung ihre Existenzberechtigung in der modernen Pädiatrie immer noch - und wider besseren Wissens - absprechen wollen. Hier hat der Vorsitzende einer großen manualmedizinischen Fachgesellschaft „danebengegriffen“. Den jüngeren Kollegen bleibt zu raten, den Weg des Konsenses weiter zu gehen - ohne den kollegialen Diskurs zu verlassen.

Im Übrigen hätten $\mathrm{m}$. E. in dieser Reihe auch Albert Cramer und Karl Sell einen würdigen Platz verdient.

H.M. Bullinger

\section{Korrespondenzadresse}

H.M. Bullinger

Freistuhl 3, 44137 Dortmund

post@manmed.info 